\title{
Triglyceride glucose index for the detection of asymptomatic coronary artery stenosis in patients with type 2 diabetes
}

Pham Viet Thai ${ }^{1}$, Hoang Anh Tien², Huynh Van Minh ${ }^{2}$ and Paul Valensi $3^{3^{*}}$

\begin{abstract}
Background: Triglyceride Glucose (TyG) index has been associated with an increased risk in cardiovascular events. Silent coronary disease is common in patients with type 2 diabetes. In Vietnam, a low-middle income country, the burden of cardiovascular disease is growing simultaneously with the epidemiologic transition. Our aim was to assess the prevalence of coronary stenoses (CS) in patients with type 2 diabetes and no history or symptom of cardiovascular disease and to investigate the association between TyG index and cardiovascular risk factors and both the presence and severity of CS. Futhermore, we assessed the value of TyG index in predicting subclinical CS.
\end{abstract}

Methods: This was a cross-sectional observational study. We recruited 166 patients at Ninh Thuan General Hospital, Vietnam. TyG index and HOMA-IR were calculated, and a coronary computed tomography angiography (CCTA) was performed.

Results: The population was classified according to tertiles of TyG index. The highest TyG values were associated with higher BMI, waist circumference, total cholesterol, LDL-cholesterol, triglycerides, plasma glucose, HbA1c levels and HOMA-IR, lower HDL-cholesterol, a higher incidence of metabolic syndrome and less frequent physical activity $(p<0.05$ to $<0.0001)$. TyG index correlated with logHOMA-IR $(p<0.0001)$. CS $\geq 50 \%$ were present in 60 participants and 32 had coronary artery stenosis $\geq 70 \%$. TyG index and HOMA-IR were significantly higher in patients with $\mathrm{CS} \geq 70 \%$. The number of narrowed coronary arteries and the degree of stenosis were associated with higher TyG index levels ( $p=0.04$ and $<0.005$ respectively). A TyG index $\geq 10$ was significantly associated with an increased risk of multiple coronary artery disease and of more severe CS. After adjusting for confounding factors, including logHOMA$I R$, these risks remained mostly significant. A TyG index threshold at 10 resulted in $57 \%$ sensitivity and $75 \%$ specificity for predicting the presence of $C S \geq 70 \%$. In subgroup analysis TyG index $\geq 10$ was associated with an increased risk in CS $\geq 70 \%$ in patients treated with statin or antiplatelet therapy.

Conclusion: More than one third of asymptomatic patients with type 2 diabetes had significant CS on CCTA. TyG index may be considered as a marker for insulin resistance and increased TyG index could identify patients with high risk of coronary artery stenoses and is associated with the number and the severity of artery stenoses.

Keywords: Triglyceride glucose index, Coronary artery disease, Type 2 diabetes, Insulin resistance, Metabolic syndrome, Coronary computed tomography angiography

\footnotetext{
*Correspondence: paul.valensi@aphp.fr

${ }^{3}$ Unit of Endocrinology-Diabetology-Nutrition, Jean Verdier Hospital,

APHP, Paris Nord University, CINFO, CRNH-IdF, Avenue du 14 Juillet, 93143 Bondy cedex, France

Full list of author information is available at the end of the article
}

\section{Background}

Diabetes is one of the major risk factors for coronary artery disease [1]. It can be estimated that $20-30 \%$ of patients with coronary disease have known diabetes, and that an oral glucose tolerance test would 
diagnose diabetes or prediabetes in up to $70 \%$ of the other patients with coronary artery disease. Actually, patients with diabetes are at substantially increased risk of fatal and non fatal coronary events [2].

In people with diabetes, coronary disease is often silent, i.e. without any cardiac symptom. In asymptomatic patients with diabetes, the prevalence of silent myocardial ischemia as detected by a stress test varies, depending on several factors including the diagnostic test and whether or not cardiac imaging is used. Furthermore, the number of additional cardiovascular risk factors and the presence of diabetic complications, especially nephropathy and cardiac autonomic neuropathy play a role $[3,4]$. The prevalence of silent ischemia which was $20-30 \%$ in the 1990's [4] has dropped in the last decade [5]. Some recent studies showed that coronary computed tomography angiography (CCTA) may detect coronary artery stenoses in a large proportion of asymptomatic patients with diabetes [6,7].

Insulin resistance is one of the pivotal risk factors for cardiovascular disease together with hyperglycemia, lipid disorders, hypertension and the production of clotting and inflammatory factors that may promote atherothrombotic changes. An independent association was reported between insulin resistance (assessed by HOMA-IR) and cardiovascular events both in subjects with and without diabetes [8-10]. In addition, several studies provide compelling evidence of an association between insulin resistance and subclinical vascular injury involving functional and structural damage to the arterial wall that is not explained by traditional risk factors [11]. Early atherosclerosis detected by intimamedia thickness is also independently associated with insulin resistance in patients with type 2 diabetes [12]. Some studies have shown that the Triglyceride Glucose index (TyG) easily calculated by using fasting glucose and triglyceride levels may be used as a surrogate for insulin resistance [13-15]. In addition TyG index was shown to predict cardiovascular events [16] and to be a marker of subclinical atherosclerosis [17]. In a large population of individuals without conventional risk factors, TyG index appeared as an independent marker for predicting subclinical coronary artery disease defined as the presence of any plaque on coronary computed tomographic angiography (CCTA) [18]. A recent Korean study reported an association between TyG index value and an increased risk of coronary artery stenoses on CCTA in patients with type 2 diabetes [7]. However, this was not confirmed in another Korean study [19]. Therefore, there was a need for further studies assessing the role of insulin resistance in the occurrence of coronary artery stenoses and the severity of coronary artery disease and the usefulness of TyG index as a marker of subclinical coronary artery disease in patients with type 2 diabetes.

Vietnam is a low-middle income country, which is undergoing an important epidemiological transition. The prevalence of Vietnamese people with multiple comorbidities has been increasing [20] with a rapid rise in the overall morbidity and mortality from noncommunicable diseases over the last two decades. The prevalence of silent coronary artery disease in the population with diabetes is expected to be greater than in higher income countries as a result of poorer control of cardiovascular risk factors.

The aim of this study performed in Vietnam was to assess the prevalence of coronary artery stenoses (CS) on CCTA and to investigate the association between TyG index and cardiovascular risk factors as well as the presence and severity of CS in patients with type 2 diabetes and no symptom or history of cardiovascular disease; the value of TyG index in predicting subclinical CS was also assessed.

\section{Methods \\ Study population and design}

This was a cross-sectional observational study. Enrolled participants were consecutive individuals diagnosed with type 2 diabetes according to the American Diabetes Association criteria [21], who were examined at Ninh Thuan General Hospital from April 2017 to May 2018. We excluded patients with type 1 diabetes and patients with an history or symptoms of cardiovascular disease such as myocardial infarction, congestive heart failure, arrhythmia or coronary revascularization, angina, edema or palpitations.

The study protocol was approved by the local ethic committee of Hue University of Medicine and Pharmacy. All patients provided written informed consent.

For each patient, medical history, physical activity, diabetes medications, treatment with statin or antiplatelet drugs was extracted from medical charts. Blood pressure was measured three times at approximately 2-min intervals after $5 \mathrm{~min}$ of seated rest, using an electronic sphygmomanometer (Omron HEM 1712), and mean values were calculated. Height without shoes was measured to the nearest $\mathrm{cm}$ using a portable stadiometer, and body weight was measured to the nearest $0.1 \mathrm{~kg}$ using a standard scale with patients wearing light indoor clothing and no shoes (Prestige, Hardik Medi Tech, India). Waist circumference was measured in the standing position, to the nearest $\mathrm{cm}$, using a measuring tape (Prestige) placed mid-distance between the costal edge and the iliac crest. 


\section{Biological measurements}

Blood samples were collected after an overnight fast and analyzed in the hospital central laboratory. Biochemical assays were immediately processed. Plasma glucose, HbA1c, total cholesterol, triglycerides, HDLcholesterol, and insulin were measured using HumaStar 600, Germany. LDL-cholesterol was calculated using Friedwald formula, and non-HDL cholesterol by subtracting HDL-cholesterol from total cholesterol. Glomerular filtration rate was estimated (eGFR) using CKD-EPI formula.

Two indicators of insulin resistance were calculated: TyG index $=$ Ln [Triglyceride $(\mathrm{mg} / \mathrm{dL}) \times$ Glucose $(\mathrm{mg} / \mathrm{dL}) / 2$ ] [13] and homeostatic model assessment of insulin resistance (HOMA-IR) index (Insulin $(\mathrm{mU} / \mathrm{L}) \times$ plasma glucose $(\mathrm{mmol} / \mathrm{L}) / 22.5)$ [22]. Metabolic syndrome was diagnosed according to NCEP-ATP III criteria [23].

\section{Coronary computed tomography angiography imaging}

Imaging was obtained by using a 64-slice scanner (Optima CT660, GE Healthcare, USA). According to the Society of Cardiovascular Computed Tomography 2014 guidelines [24], a 16-segment coronary artery tree model was used. All patients had normal sinus rhythm. If heart rate was faster than $70 \mathrm{bpm}$, an oral dose of 50-100 mg of metoprolol was administered 60-90 min before the study. If this did not lower the heart rate to the desired level, 5-mg doses of intravenous metoprolol were administered at 3- to 5-min intervals, up to a total dose of 15-30 mg. Images were obtained before and after administration of iodinated contrast (iopromide). Percent diameter stenosis was measured at the minimal lumen area (detected lumen contours), and corresponding reference diameter values were obtained from an automatic trend analysis of the vessel areas within the artery. The principles of coronary artery lesion interpretation included (1) systematic review of each coronary segment from multiple slices and in transverse sections, (2) awareness of relevant artifacts, and (3) assessment of stenosis severity using high resolution images (including multiplanar reformation format) in both longitudinal and transverse views of the artery. An image review in the frontal and lateral views could aid in the identification of artifacts. One specialist experienced in image reading reviewed the arterial tree in detail beginning in the axial (caudal) view since less processed trans-axial data are more robust. CS was considered as significant when $\geq 50 \%$ in one or more main branch of coronary arteries, moderate from 50 to $69 \%$ and severe if $\geq 70 \%$.

\section{Statistical analyses}

The sample size was calculated considering an estimated proportion of patients with significant coronary stenoses of $12 \%$ and a confidence level of $95 \%$, leading to a minimum sample size of 162 patients.

The normality of the data was assessed using the Kolmogorov-Smirnov test. Results were expressed as mean $\pm \mathrm{SD}$, median (interquartile range) or percentages. Between group comparisons for quantitative parameters were performed using one-way ANOVA test or KruskallWallis test. Between group comparisons for categorical parameters were performed by chi square tests. Correlations between quantitative parameters were performed by using Pearson test. ROC curve analysis was used to evaluate the accuracy of TyG index in detecting metabolic syndrome and CS. Multinomial logistic regression analyses were performed in order to assess the association of TyG index with CS after adjustment for confounding factors. Odds ratios with 95\% confidence intervals ( $95 \%$ CI) for the risk of CS are reported. Subgroup analysis was performed after separating the patients according to statin and antiplatelet use. A p value $<0.05$ was considered significant for all analyses. Statistical analyses were carried out using SPSS (Statistical Product and Services Solutions) software version 20.0.

\section{Results \\ Main clinical characteristics of the study population}

A total of 166 consecutive patients with type 2 diabetes were included: $62 \%$ male, mean age $58.9 \pm 10.8$ years, BMI $24.8 \pm 2.6 \mathrm{~kg} / \mathrm{m}^{2}$, HbA1c $7.9 \pm 1.0 \%$. TyG index mean value was $9.64 \pm 0.63$ (range: $7.80-10.96$ ).

\section{Clinical and biochemical characteristics according to TyG index tertiles}

We classified the population into tertiles of TyG index. In patients with higher TyG indexes, BMI, waist circumference, total cholesterol, triglycerides, LDL-cholesterol, non-HDL cholesterol, plasma glucose, HbA1c, insulin and HOMA-IR levels were higher whereas HDL-cholesterol levels and the frequency of physical activity were lower. There was no significant difference for the current treatments across TyG tertiles (Table 1).

\section{Association of TyG index with metabolic syndrome and HOMA-IR index}

Metabolic syndrome was present in 109 patients (65.7\%). The percentage of patients with metabolic syndrome was higher in the middle and high TyG index tertiles (Table 1). TyG index was significantly associated with metabolic syndrome with an area under the ROC curve at 0.745 (95\% CI: 0.660-0.830, p < 0.001) 
Table 1 Clinical and biological characteristics according to the TyG tertiles

\begin{tabular}{|c|c|c|c|c|}
\hline & $\begin{array}{l}\text { Lowest tertile }(7.80-9.37) \\
(n=56)\end{array}$ & $\begin{array}{l}\text { Mid tertile (9.38-9.99) } \\
(\mathrm{n}=55)\end{array}$ & $\begin{array}{l}\text { Highest tertile }(10.00- \\
10.96)(n=55)\end{array}$ & p value \\
\hline Age (years) & $58.5 \pm 10.1$ & $60.6 \pm 11.4$ & $57.5 \pm 11.0$ & 0.306 \\
\hline Gender (M/F) & $36 / 20$ & $34 / 21$ & $33 / 22$ & 0.897 \\
\hline BMI $\left(\mathrm{kg} / \mathrm{m}^{2}\right)$ & $23.6 \pm 2.8$ & $25.0 \pm 2.5$ & $25.8 \pm 2.1$ & $<0.001$ \\
\hline Waist circumference (cm) & $86.0 \pm 11.7$ & $91.2 \pm 10.9$ & $94.0 \pm 9.9$ & 0.001 \\
\hline Male & $90.2 \pm 11.9$ & $94.9 \pm 9.9$ & $96.4 \pm 9.1$ & 0.041 \\
\hline Female & $78.4 \pm 6.1$ & $85.2 \pm 10.2$ & $90.4 \pm 10.2$ & $<0.001$ \\
\hline Systolic blood pressure (mmHg) & $138.9 \pm 21.3$ & $139.2 \pm 22.9$ & $143.7 \pm 23.1$ & 0.456 \\
\hline Diastolic blood pressure $(\mathrm{mmHg})$ & $85.9 \pm 13.4$ & $85.4 \pm 14.1$ & $85.6 \pm 14.0$ & 0.975 \\
\hline Duration of diabetes (years) & $4[3-6]$ & $4[3-8]$ & $7[3-12]$ & 0.044 \\
\hline Practicing physical activity & $24(42.9 \%)$ & $12(21.8 \%)$ & $7(12.7 \%)$ & 0.001 \\
\hline Smoking & $15(26.8 \%)$ & $22(40 \%)$ & $24(43.6 \%)$ & 0.152 \\
\hline Metabolic syndrome & $23(41.1 \%)$ & $42(76.4 \%)$ & $44(80.0 \%)$ & $<0.001$ \\
\hline Total cholesterol (mg/dL) & $207.1 \pm 46.3$ & $224.1 \pm 55.2$ & $232.8 \pm 52.7$ & 0.03 \\
\hline Triglycerides (mg/dL) & $146.9 \pm 42.2$ & $209.9 \pm 44.2$ & $330.8 \pm 70.4$ & $<0.001$ \\
\hline HDL-cholesterol (mg/dL) & $50.0 \pm 12.4$ & $41.9 \pm 8.2$ & $40.1 \pm 7.8$ & $<0.001$ \\
\hline LDL-cholesterol (mg/dL) & $126.1 \pm 36.7$ & $130.4 \pm 44.0$ & $146.0 \pm 51.5$ & 0.042 \\
\hline Non-HDL cholesterol (mg/dl) & $157.1 \pm 43.3$ & $182.2 \pm 54.6$ & $192.7 \pm 51.5$ & $<0.001$ \\
\hline Fasting plasma glucose (mg/dL) & $111.9 \pm 27.7$ & $159.7 \pm 29.3$ & $189.0 \pm 43.2$ & $<0.001$ \\
\hline $\mathrm{HbA1c}(\%)$ & $7.3 \pm 0.6$ & $8.0 \pm 0.9$ & $8.3 \pm 1.0$ & $<0.001$ \\
\hline TyG index & $8.95 \pm 0.37$ & $9.67 \pm 0.19$ & $10.33 \pm 0.23$ & $<0.001$ \\
\hline Insulin (mU/L) & $17[12-28]$ & 30 [18-39] & 30 [18-39] & $<0.001$ \\
\hline HOMA-IR index & $4.45[2.85-6.88]$ & $10.20[6.40-14.30]$ & $12.40[8.30-16.60]$ & $<0.0001$ \\
\hline $\mathrm{eGFR}\left(\mathrm{mL} / \mathrm{min} / 1.73 \mathrm{~m}^{2}\right)$ & $89.4 \pm 28.1$ & $82.2 \pm 21.0$ & $84.0 \pm 20.3$ & 0.239 \\
\hline Coronary artery stenosis $\geq 50 \%(\%)$ & $16(28.6)$ & $16(29.1)$ & $28(50.9)$ & 0.021 \\
\hline Coronary artery stenosis $\geq 70 \%(\%)$ & $5(8.9)$ & $11(20.0)$ & $16(29.1)$ & 0.026 \\
\hline \multicolumn{5}{|l|}{ Drug therapy } \\
\hline Metformin & $55(98.2 \%)$ & $53(96.4 \%)$ & $55(100 \%)$ & 0.359 \\
\hline Sulphonylurea & $50(89.3 \%)$ & $51(92.7 \%)$ & 48 (87.3\%) & 0.634 \\
\hline Thiazolidinedione & $1(1.8 \%)$ & $0(0 \%)$ & $0(0 \%)$ & 0.372 \\
\hline DPP-4 inhibitors & $1(1.8 \%)$ & $1(1.8 \%)$ & $3(5.5 \%)$ & 0.432 \\
\hline a-glucosidase inhibitor & $4(7.1 \%)$ & $12(21.8 \%)$ & $14(25.5 \%)$ & 0.029 \\
\hline Insulin & $2(3.6 \%)$ & $0(0 \%)$ & $1(1.8 \%)$ & 0.369 \\
\hline Antiplatelet & $30(53.6 \%)$ & $32(58.2 \%)$ & $32(58.2 \%)$ & 0.852 \\
\hline Statin & 33 (58.9\%) & $32(58.2 \%)$ & $31(56.4 \%)$ & 0.961 \\
\hline
\end{tabular}

Data are expressed as mean $\pm S D$, median (interquartile range) or number (\%)

Comparisons were performed using ANOVA or Kruskall-Wallis tests and chi square tests $B M I$ body mass index, M/F male/female, eGFR estimated glomerular filtration rate, HOMA-IR homeostatic model assessment of insulin resistance, TyG triglyceride glucose index

(Fig. 1). A TyG index threshold at 9.145 resulted in a 93.6\% sensitivity and a $52.6 \%$ specificity; positive and negative predictive values were respectively $77.3 \%$ and 79.4\%. There was a significant correlation between TyG index and $\log$ HOMA-IR index $(r=0.644, p<0.0001)$ (Fig. 2).

\section{Results of coronary computed tomography angiography} and association with TyG index

In the total population $(\mathrm{n}=166), 60$ participants had $\mathrm{CS} \geq 50 \%$, including 32 with $\mathrm{CS} \geq 70 \%$. Twenty-one patients had 2- or 3-vessel disease (with stenosis $\geq 50 \%$ ). 


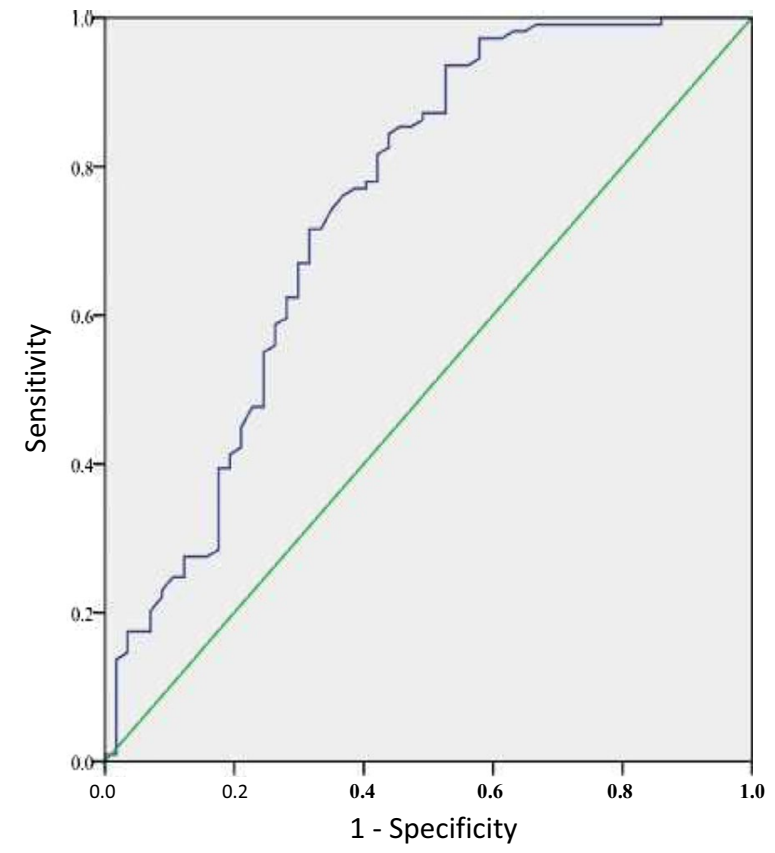

Fig. 1 ROC curve of the use of TyG index in the detection of metabolic syndrome

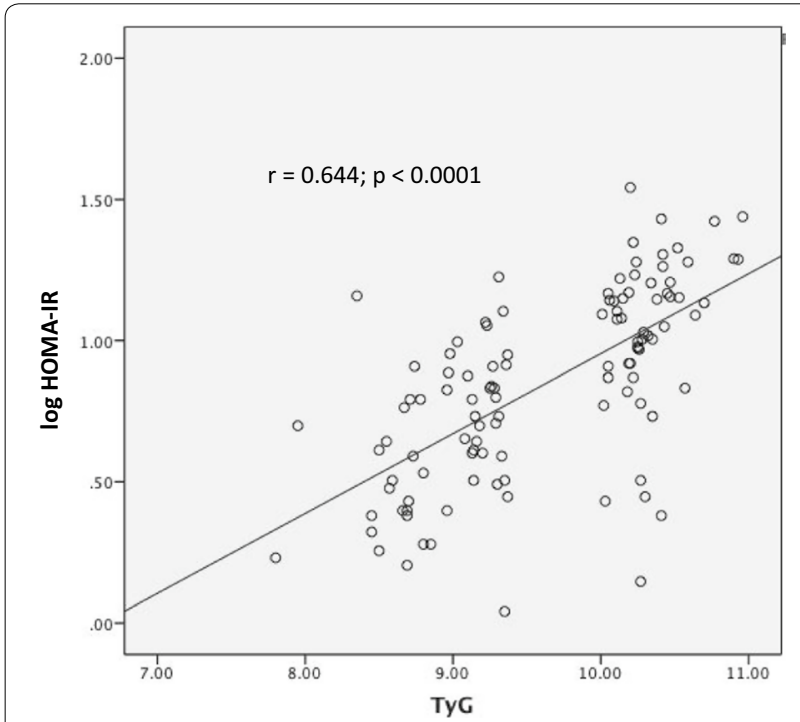

Fig. 2 Correlation between TyG index and log HOMA-IR index

Compared to patients without significant CS, those with $C S \geq 50 \%$ were older, had longer diabetes duration, higher BMI, blood pressure and HbA1c, had more often been diagnosed with metabolic syndrome, were more frequently smokers, and were more often treated with statin or antiplatelet therapy. TyG index and HOMA-IR were significantly higher in patients with $\mathrm{CS} \geq 70 \%$ (Table 2).

The prevalence of significant CS did not differ across the tertiles of HOMA-IR (30.9, 32.1 and 45.5\%, from the lowest to the highest tertile; $\mathrm{p}=0.211$ ). The prevalence of $\mathrm{CS} \geq 50 \%$ and $\geq 70 \%$ were higher in the highest TyG index tertile subgroup versus the lowest and middle tertile subgroups (respectively, 50.9 vs 28.6 and $29.1 \%, \mathrm{p}=0.021$; and 29.1 vs 8.9 and $20 \%, \mathrm{p}=0.026$ ) (Table 1). TyG index significantly predicted the presence of $\mathrm{CS} \geq 70 \%$ with an area under the ROC curve at 0.678 (95\% CI: 0.582-0.775, $\mathrm{p}=0.002$ ). A TyG index threshold at 10 gave $57 \%$ sensitivity, $75 \%$ specificity, $56 \%$ positive predictive value and $75 \%$ negative predictive value.

The number of stenosed coronary arteries $(\mathrm{p}=0.04)$ and the degree of CS $(p<0.005)$ were positively associated with the TyG index level (Fig. 3a, b). TyG index $\geq 10$ was significantly associated with an increased risk of having a higher number of stenosed arteries and more severe coronary artery stenoses. After adjusting for factors significantly associated with the severity of CS, including logHOMA-IR, these risks remained mostly significant (Table 3).

Subgroup analyses showed that TyG index $\geq 10$ was associated with an increased risk of $\mathrm{CS} \geq 70 \%$ in patients on statin or antiplatelet therapy but not in those not taking these treatments (Fig. 4).

\section{Discussion}

In this study we included patients with type 2 diabetes and no symptom or history of cardiovascular disease but with rather poor control of hypertension and lipid disorders. TyG index correlated with HOMA-IR. Patients with higher TyG index had more metabolic disorders. More than one third of our patients had coronary stenoses on CCTA and $19 \%$ had severe CS ( $\geq 70 \%)$. Patients with severe CS had higher TyG index and HOMA-IR. The prevalence of $C S \geq 50 \%$ and $\geq 70 \%$ was higher in the high tertile of TyG index but did not differ across the tertiles of HOMA-IR. TyG index significantly predicted the presence of $C S \geq 70 \%$. In addition, we showed for the first time in an asymptomatic population that the number of stenosed coronary arteries and the degree of coronary stenosis were associated with high TyG index levels. TyG index $\geq 10$ was significantly associated with a 3 - to 5 -fold greater risk of higher number of stenosed coronary arteries and of more severe coronary stenoses, and these risks remained mostly significant after adjustment for confounding factors. Our results suggest that in patients with type 2 diabetes TyG index may be considered as a marker of insulin resistance and could identify patients with high coronary risk. 
Table 2 Comparison between the patients without significant coronary artery stenosis (CS) or with moderate (50-69\%) or severe $(\geq 70 \%)$ CS

\begin{tabular}{|c|c|c|c|c|c|}
\hline & Total & No significant CS & CS 50-69\% & $\mathrm{CS} \geq 70 \%$ & $\mathbf{P}$ \\
\hline Number & 166 & 106 & 28 & 32 & \\
\hline Age (years) & $58.9 \pm 10.8$ & $57.2 \pm 10.7$ & $60.9 \pm 9.8$ & $62.8 \pm 11.0$ & 0.020 \\
\hline Gender (M/F) & $103 / 63$ & $63 / 43$ & $21 / 7$ & $19 / 13$ & 0.301 \\
\hline BMI $\left(\mathrm{kg} / \mathrm{m}^{2}\right)$ & $24.8 \pm 2.6$ & $24.5 \pm 2.7$ & $24.6 \pm 2.5$ & $26.0 \pm 2.1$ & 0.019 \\
\hline Waist circumference (cm) & $90.4 \pm 11.3$ & $88.8 \pm 11.3$ & $92.1 \pm 11.7$ & $94.0 \pm 10.4$ & 0.054 \\
\hline Systolic blood pressure (mmHg) & $140.6 \pm 22.4$ & $130.3 \pm 18.5$ & $152.0 \pm 18.3$ & $165.0 \pm 11.1$ & $<0.0001$ \\
\hline Diastolic blood pressure $(\mathrm{mmHg})$ & $85.7 \pm 13.8$ & $80.3 \pm 11.2$ & $91.1 \pm 14.9$ & $98.8 \pm 9.5$ & $<0.0001$ \\
\hline Duration of diabetes (years) & $4.00[3.00-8.00]$ & $3.00[2.00-4.00]$ & $7.00[6.00-9.00]$ & $11.50[8.00-12.00]$ & $<0.0001$ \\
\hline Practicing physical activity & $43(25.9)$ & $30(28.3)$ & $10(35.7)$ & $3(9.4)$ & 0.043 \\
\hline Smoking & $61(36.7)$ & $27(25.5)$ & $15(53.6)$ & $19(59.4)$ & $<0.0001$ \\
\hline Metabolic syndrome & $109(65.7)$ & $60(56.6)$ & $17(60.7)$ & $32(100.0)$ & $<0.0001$ \\
\hline Total cholesterol (mg/dl) & $221.2 \pm 52.3$ & $222.7 \pm 53.3$ & $225.5 \pm 46.1$ & $212.5 \pm 54.8$ & 0.562 \\
\hline Triglycerides (mg/dl) & $228.7 \pm 93.4$ & $218.3 \pm 94.3$ & $233.6 \pm 98.6$ & $259.0 \pm 80.4$ & 0.091 \\
\hline HDL-cholesterol (mg/dl) & $44.0 \pm 10.6$ & $45.2 \pm 11.3$ & $44.2 \pm 10.8$ & $40.0 \pm 6.4$ & 0.053 \\
\hline LDL-cholesterol (mg/dl) & $134.3 \pm 45.0$ & $130.5 \pm 44.9$ & $143.2 \pm 51.5$ & $139.2 \pm 39.0$ & 0.330 \\
\hline Non-HDL cholesterol (mg/dl) & $177.2 \pm 51.9$ & $177.5 \pm 52.7$ & $181.3 \pm 47.7$ & $172.5 \pm 54.0$ & 0.804 \\
\hline Fasting plasma glucose (mg/dl) & $153.6 \pm 46.8$ & $147.0 \pm 43.5$ & $148.2 \pm 53.3$ & $180.2 \pm 43.0$ & 0.001 \\
\hline HbA1c (\%) & $7.9 \pm 1.0$ & $7.6 \pm 0.8$ & $7.9 \pm 0.8$ & $8.9 \pm 1.1$ & 0.000 \\
\hline TyG index & $9.64 \pm 0.63$ & $9.56 \pm 0.61$ & $9.58 \pm 0.71$ & $9.97 \pm 0.49$ & 0.005 \\
\hline Insulin (mU/L) & $22[15-34]$ & $21[14-35]$ & 20 [12-31] & $30[20-40]$ & 0.092 \\
\hline HOMA-IR index & $8.10[4.65-13.93]$ & $7.60[4.33-12.83]$ & $6.20[2.95-11.90]$ & $13.45[7.48-17.53]$ & 0.001 \\
\hline $\mathrm{eGFR}\left(\mathrm{mL} / \mathrm{min} / 1.73 \mathrm{~m}^{2}\right)$ & $85.3 \pm 23.5$ & $88.7 \pm 26.0$ & $80.2 \pm 15.8$ & $78.3 \pm 18.2$ & 0.040 \\
\hline \multicolumn{6}{|l|}{ Drug therapy } \\
\hline Metformin & $163(98.2)$ & $104(98.1)$ & $28(100)$ & $31(96.9)$ & 0.660 \\
\hline Sulphonylurea & $149(89.8)$ & $91(85.8)$ & $28(100)$ & $30(93.8)$ & 0.064 \\
\hline Thiazolidinedione & $1(0.6)$ & $1(0.9)$ & $0(0)$ & $0(0)$ & 0.752 \\
\hline DPP-4 inhibitor & $5(3.0)$ & $0(0)$ & $2(7.1)$ & $3(9.4)$ & 0.009 \\
\hline a-glucosidase inhibitor & $30(18.1)$ & $5(4.7)$ & $9(32.1)$ & $16(50.0)$ & $<0.0001$ \\
\hline Insulin & $3(1.8)$ & $0(0)$ & $2(7.1)$ & $1(3.1)$ & 0.034 \\
\hline Antiplatelet & $94(56.6)$ & $46(43.4)$ & $23(82.1)$ & $25(78.1)$ & $<0.0001$ \\
\hline Statin & $96(57.8)$ & $47(44.3)$ & $23(82.1)$ & $26(81.2)$ & $<0.0001$ \\
\hline
\end{tabular}

Data are expressed as mean $\pm S D$, median (interquartile range) or number (\%)

Comparisons were performed using ANOVA or Kruskall-Wallis tests and chi square tests

$B M I$ body mass index, CS coronary stenosis, eGFR estimated glomerular filtration rate, HOMA-IR homeostatic model assessment of insulin resistance, $M / F$ male/female, TyG index triglyceride glucose index

\section{TyG index as a marker of insulin resistance and metabolic syndrome}

TyG index, a composite indicator based on triglyceride level and fasting plasma glucose value, was shown to correlate with insulin resistance as assessed by hyperinsulinemic euglycemic clamp or HOMA-IR [13-15] and may thus be used as a surrogate marker of insulin resistance. When compared to clamp, TyG index could even perform better than HOMA-IR [25]. TyG index was also reported to be a marker of metabolic disorders $[7,14,26]$ and a good predictor of incident type 2 diabetes [27-29]. In our population of patients with type 2 diabetes, higher TyG index was strongly associated with higher HOMA-IR and more pronounced metabolic disorders including higher BMI, increased waist circumference, total cholesterol, triglycerides, LDL-cholesterol, non-HDL cholesterol, plasma glucose and HbA1c levels, lower HDL-cholesterol levels and also less physical activity. This index was not associated with current glucoselowering treatments except for $\alpha$-glucosidase inhibitors (only 30 patients on this treatment) nor with lipid lowering treatment, noting that none of our patients was on fibrate, which might have altered TyG index. Interestingly, TyG index calculation effectively led to metabolic 

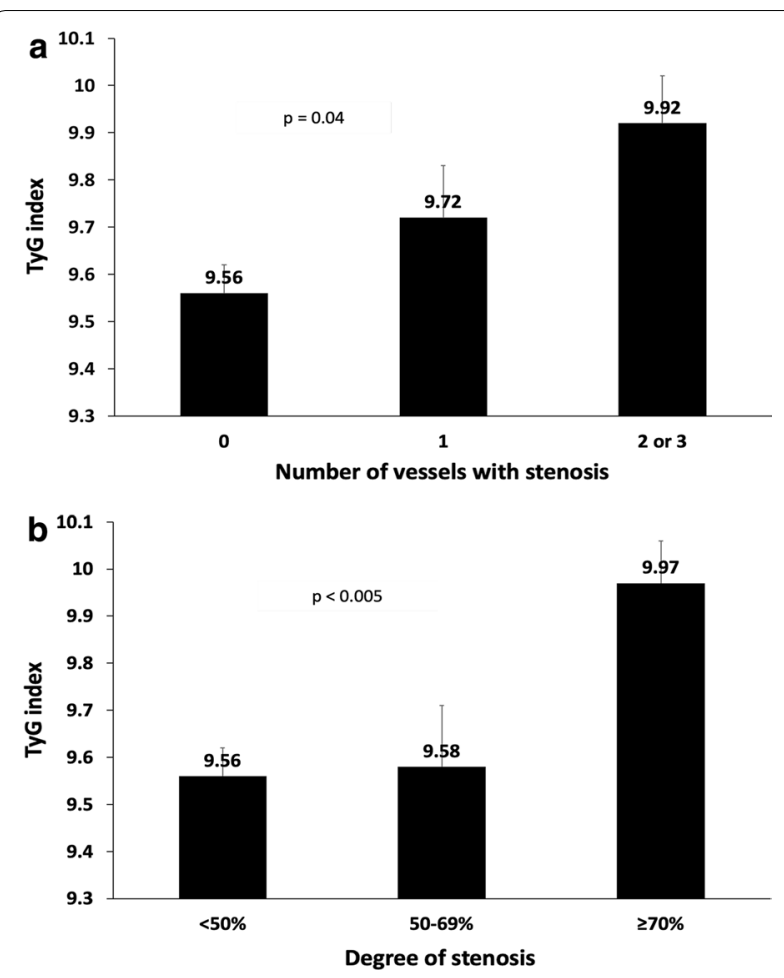

Fig. 3 TyG index according to the number of coronary arteries with stenosis $\geq 50 \%$ (Part A) and the severity of coronary stenosis (Part B)

syndrome diagnosis with rather good performances when using a threshold at 9.145.

TyG index as a marker of atherosclerosis and cardiovascular complications

Several studies suggest that TyG index might be recognized as a risk factor for cardiovascular complications and a marker of atherosclerosis. TyG index was shown to be an independent predictor of cardiovascular events in a healthy population [16] and to be associated with a higher incidence of cardiovascular outcomes in patients with stable coronary artery disease [30] including those with type 2 diabetes [31], in patients with non-ST elevation acute coronary syndrome [32] and in those with acute ST-elevation myocardial infarction after percutaneous coronary intervention [33]. This index was reported to be a better marker than HOMA-IR for subclinical carotid atherosclerosis and arterial stiffness in the general population [34-36] and in lean postmenopausal women [17]. In a healthy population TyG index was more independently associated with coronary artery calcifications than was HOMA-IR [26]. Elevated TyG index was also shown to be an independent predictor of progression of coronary calcifications [37].

\section{Cardiovascular risk in contemporary South-Asian countries}

Cardiovascular disease is becoming a major cause of deaths in Vietnam like in other low-middle income countries. In the setting of acute myocardial infarction, the incidence of in-hospital death rates is higher in patients with multiple cardiac comorbidities [38] with a need to improve guideline adherence [39]. The proportion of patients with diabetes and silent coronary artery disease is expected to be greater than in higher income countries. In the present study, CCTA detected coronary stenoses $\geq 50$ in $36 \%$ and $\geq 70$ in $19 \%$ of the patients. In two previous studies which similarly performed CCTA in asymptomatic individuals, the proportion of patients with CS $\geq 70 \%$ was found lower: $6.3 \%$ in a US population of patients with type 1 or type 2 diabetes considered to be at high cardiovascular risk [6] and $12.3 \%$ in a Korean population of patients with type 2 diabetes [7]. Compared to the latter study, our population had some similarities but the risk was greater because blood pressure, triglycerides and LDL-cholesterol levels were higher, and

Table 3 Association between TyG index $\geq 10$ and the number of coronary arteries with stenosis $\geq 50 \%$ and the degree of coronary stenosis

\begin{tabular}{|c|c|c|c|c|c|c|}
\hline & Unadjusted OR (95\% Cl) & p value & Model 1 OR $(95 \% \mathrm{Cl})$ & $p$ value & Model 2 OR $(95 \% \mathrm{Cl})$ & $p$ value \\
\hline \multicolumn{7}{|c|}{ Number of vessels with stenosis } \\
\hline 0 & 1 & & 1 & & 1 & \\
\hline 1 & $3.77(1.76-8.17)$ & 0.001 & $4.95(1.52-16.09)$ & 0.008 & $6.88(1.94-24.38)$ & 0.003 \\
\hline 2 or 3 & $3.90(1.48-10.28)$ & 0.006 & $3.02(0.75-12.21)$ & 0.121 & $2.74(0.64-11.76)$ & 0.176 \\
\hline \multicolumn{7}{|c|}{ Degree of coronary stenosis } \\
\hline$<50 \%$ & 1 & & 1 & & & \\
\hline $50-69 \%$ & $2.93(1.24-6.92)$ & 0.014 & $4.86(1.39-17.03)$ & 0.013 & $6.89(1.80-26.44)$ & 0.005 \\
\hline$\geq 70 \%$ & $4.88(2.11-11.28)$ & 0.0001 & $4.36(1.15-16.53)$ & 0.030 & $4.04(1.00-16.34)$ & 0.050 \\
\hline
\end{tabular}

Multinomial logistic regression analyses were performed

Model 1 Adjusted for duration of diabetes of 4 years (median value in the total population), body mass index, estimated glomerular filtration rate, practicing physical activity, smoking, $\mathrm{HbA} 1 \mathrm{c}$ of $7.9 \%$ and systolic blood pressure of $140 \mathrm{mmHg}$ (mean values in the total population), Model 2 Model $1+$ adjusted for log HOMA-IR, OR odds ratio, $\mathrm{Cl}$ confidence interval 


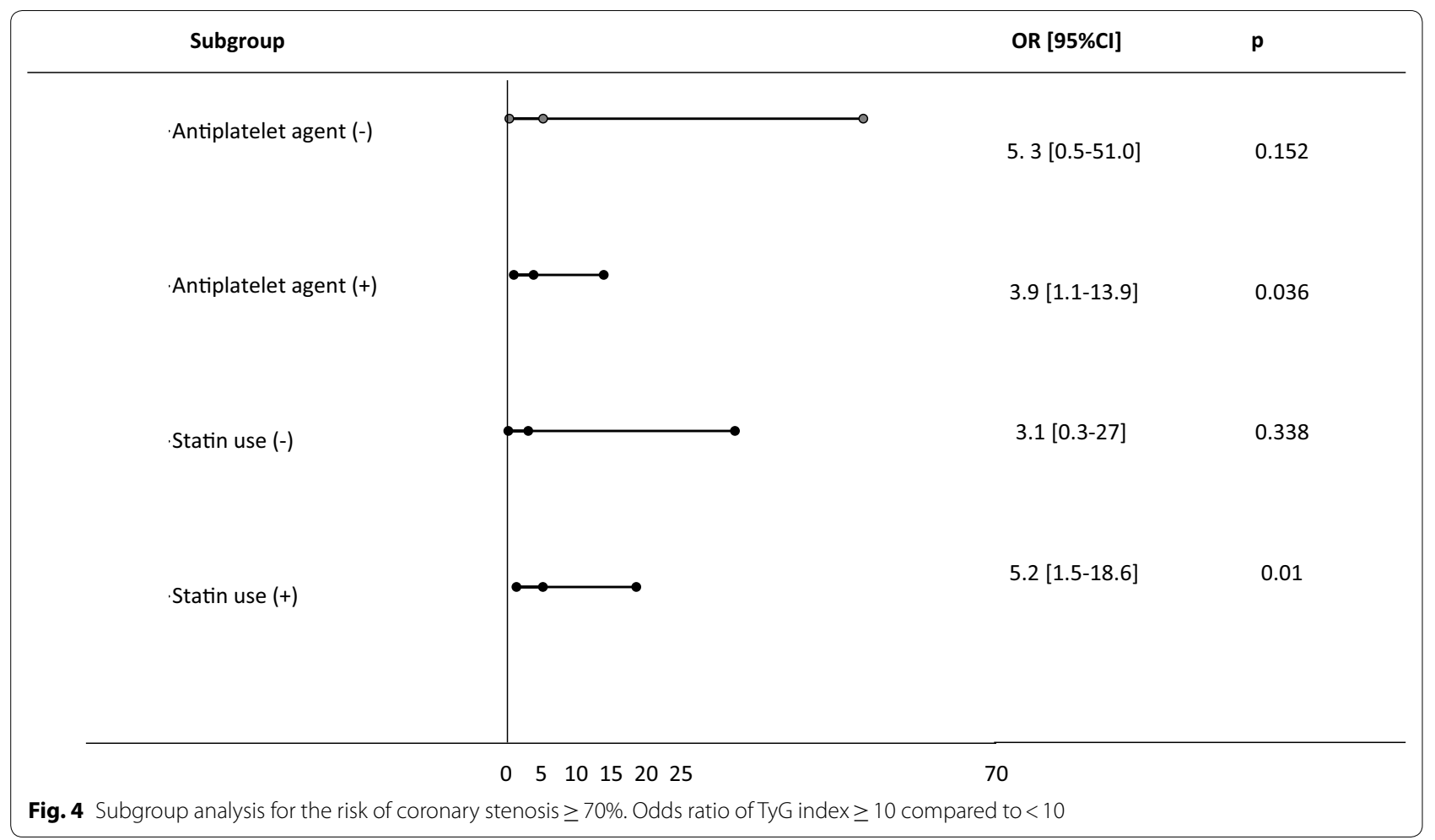

TyG index was also higher. The levels of these risk factors were clearly above target values according to the recent guidelines [1].

\section{TyG index is an independent marker of the severity of silent coronary disease}

In our study, TyG index was associated with an increased risk of CS on CCTA, consistently with previous reports [7]. Additionnally, since diabetes is associated with an increase in the extension and severity of coronary artery disease, we evaluated for the first time the severity of CS and found that TyG index $\geq 10$ was associated with an increased number of stenosed vessels and more severe stenosis. This risk was independent of diabetes duration, BMI, systolic blood pressure, HbA1c, eGFR, physical activity and smoking. This result is consistent with a previous study in patients with non-ST elevation acute coronary syndrome observing an independent association of TyG index with the number of stenosed coronary arteries and the SYNTAX score [32]. This suggests that insulin resistance as expressed by TyG index may contribute to atherosclerosis in addition to cardiovascular risk factors.

However the association of TyG index with more severe coronary disease remained significant after further adjustment on logHOMA-IR, suggesting that both metabolic disorders expressed by TyG index may play a role in coronary atherosclerosis. Improved glycemic control is expected to reduce TyG index level and may contribute to reduce cardiovascular outcomes in patients with type 2 diabetes [40]. Whether or not triglycerides are involved in the atherosclerotic process is still debated. Some data strongly suggest a relation between elevated triglyceride levels and cardiovascular disease $[41,42]$ with a residual cardiovascular risk associated with triglycerides in patients with type 2 diabetes who have reached their LDL-cholesterol target [43, 44]. A major role of the retention of cholesterol-rich and triglyceride-rich apoBcontaining remnants within the arterial wall is to be considered in the pathogenesis of atherosclerosis [45].

In subgroup analyses we observed that TyG index was associated with an increased risk of $\mathrm{CS} \geq 70 \%$ in patients on statin or antiplatelet therapy. This suggests that TyG index might account at least partly for the residual risk that remains despite these treatments. Thus, triglyceride level lowering appears as an additional target in patients at high cardiovascular risk [1, 43, 46-49].

Our study has some limitations. First, its cross-sectional design prevents us from considering the results as a definite causality relationship and the mechanisms behind the association of TyG index and CS remain to be clarified. Second, our study population was relatively small. A TyG index threshold at 10 to identify patients at high risk of severe silent coronary stenosis needs to be confirmed in further studies. Third, we compared 
TyG index with HOMA-IR and not with the results of an hyperinsulinemic euglycemic clamp, the gold standard method for measuring insulin resistance. However, several studies have previously shown similar associations of TyG index with clamp test results. Fourth, we did not record any nutritional data and therefore, we were unable to adjust for diet habits that can dramatically change triglyceride levels. Fifth, the participants were recruited in one hospital center, which may limit the generalizability of our results. However, our study has a major strength, as we performed CCTA in a relatively homogeneous population of patients with type 2 diabetes who did not have any evidence of cardiovascular disease.

\section{Conclusion}

Our results strongly suggest that in patients with type 2 diabetes, TyG index may be used as a tool to assess insulin resistance and to identify patients with metabolic syndrome. This simple and inexpensive tool may be easily used in low-middle income countries.

In our population, more than one third of the participants had CS on CCTA and TyG index was better than HOMA-IR to identify patients at high risk. Higher TyG index was associated with the number of stenosed coronary arteries and the severity of stenosis independently from classical risk factors. The predictive value of TyG index seems to be additional to classical risk factors and remains significant even in patients on statin or antiplatelet therapy. Insulin resistance but also high triglyceride levels might thus be involved in the residual cardiovascular risk. Finally, TyG index might be beneficial for stratification and intervention to prevent major cardiac events. Further studies in larger populations are needed to assess the importance of these results.

\section{Abbreviations \\ CCTA: Coronary computed tomography angiography; CS: Coronary stenoses: eGFR: Estimated glomerular filtration rate; HOMA-IR: Homeostatic model assessment of insulin resistance; TyG: Triglyceride Glucose index.}

\section{Acknowledgements}

The authors are grateful to Cao Huy Tung, MD for the readings of CCTAs and Minh Tuan Nguyen, MD, PhD for his contribution to statistical analyses and thank Sylvie Picard MD, PhD for her help in the preparation of the English manuscript.

\section{Authors' contributions}

All authors have made substantial contributions. HVM conceived the study and designed the study protocol. PVT, HAT and HVM organized, performed the study investigations and supported the recruitment of the patients. HAT performed statistical analyses. PVT, HVM and PV wrote the first draft of the manuscript. All authors critically revised the manuscript for important intellectual content. All authors read and approved the final manuscript.

\section{Funding}

This research did not receive any specific grant from funding agencies in the public, commercial, or not-for-profit sectors.

\section{Availability of data and materials}

The datasets generated during and/or analysed during the current study are available from the corresponding author on reasonable request.

\section{Ethics approval and consent to participate}

The study protocol was approved by our institution's ethics committee, and informed consent for the procedure was obtained from each participant.

\section{Consent for publication}

Not applicable.

\section{Competing interests}

The authors declare they have no competing interests.

\section{Author details}

${ }^{1}$ Department of Internal Medicine, Ninh Thuan Province General Hospital, Phan Rang - Thap Cham, Vietnam. ${ }^{2}$ Department of Internal Medicine, Hue University of Medicine and Pharmacy, Hue University, Hue, Vietnam. ${ }^{3}$ Unit of Endocrinology-Diabetology-Nutrition, Jean Verdier Hospital, APHP, Paris Nord University, CINFO, CRNH-IdF, Avenue du 14 Juillet, 93143 Bondy cedex, France.

Received: 29 March 2020 Accepted: 29 August 2020

Published online: 12 September 2020

\section{References}

1. Cosentino F, Grant PJ, Aboyans V, Bailey CJ, Ceriello A, Delgado V, Federici M, Filippatos G, Grobbee DE, Hansen TB, Huikuri HV, Johansson I, Jüni P, Lettino M, Marx N, Mellbin LG, Östgren CJ, Rocca B, Roffi M, Sattar N, Seferović PM, Sousa-Uva M, Valensi P, Wheeler DC, ESC Scientific Document Group. 2019 ESC Guidelines on diabetes, pre-diabetes, and cardiovascular diseases developed in collaboration with the EASD: The Task Force for diabetes, pre-diabetes, and cardiovascular diseases of the European Society of Cardiology (ESC) and the European Association for the Study of Diabetes (EASD). Eur Heart J. 2020;41:255-323.

2. Peters SA, Huxley RR, Woodward M. Diabetes as risk factor for incident coronary heart disease in women compared with men: a systematic review and meta-analysis of 64 cohorts including 858,507 individuals and 28,203 coronary events. Diabetologia. 2014;57:1542-51.

3. Cosson E, Attali JR, Valensi P. Markers for silent myocardial ischemia in diabetes. Are they helpful? Diabetes Metab. 2005;31:205-13.

4. Valensi P, Sachs RN, Harfouche B, Lormeau B, Paries J, Cosson E, Paycha F, Leutenegger M, Attali JR. Predictive value of cardiac autonomic neuropathy in diabetic patients with or without silent myocardial ischemia. Diabetes Care. 2001;24:339-43.

5. Sultan A, Perriard F, Macioce V, Mariano-Goulart D, Boegner C, Daures JP, Avignon A. Evolution of silent myocardial ischaemia prevalence and cardiovascular disease risk factor management in Type 2 diabetes over a 10-year period: an observational study. Diabet Med. 2017;34:1244-51.

6. Muhlestein JB, Lappé DL, Lima JA, Rosen BD, May HT, Knight S, et al. Effect of screening for coronary artery disease using $\mathrm{CT}$ angiography on mortality and cardiac events in high-risk patients with diabetes: the FACTOR-64 randomized clinical trial. JAMA. 2014;312:2234-43.

7. Lee EY, Yang HK, Lee J, Kang B, Yang Y, Lee SH, et al. Triglyceride glucose index, a marker of insulin resistance, is associated with coronary artery stenosis in asymptomatic subjects with type 2 diabetes. Lipids Health Dis. 2016;15:155.

8. Bonora E, Formentini G, Calcaterra F, Lombardi S, Marini F, Zenari L, et al. HOMA-estimated insulin resistance is an independent predictor of cardiovascular disease in type 2 diabetic subjects: prospective data from the Verona Diabetes Complications Study. Diabetes Care. 2002;25:1135-41.

9. Hanley AJ, Williams K, Stern MP, Haffner SM. Homeostasis model assessment of insulin resistance in relation to the incidence of cardiovascular disease: the San Antonio Heart Study. Diabetes Care. 2002;25:1177-84.

10. Jeppesen J, Hansen TW, Rasmussen S, Ibsen H, Torp-Pedersen C, Madsbad S. Insulin resistance, the metabolic syndrome, and risk of incident cardiovascular disease: a population-based study. J Am Coll Cardiol. 2007:49:2112-9. 
11. Adeva-Andany MM, Ameneiros-Rodríguez E, Fernández-Fernández C, Domínguez-Montero A, Funcasta-Calderón R. Insulin resistance is associated with subclinical vascular disease in humans. World J Diabetes. 2019;10:63-77.

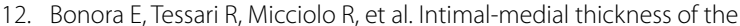
carotid artery in nondiabetic and NIDDM patients. Relationship with insulin resistance Diabetes Care. 1997;20:627-31.

13. Simental-Mendía LE, Rodríguez-Morán M, Guerrero-Romero F. The product of fasting glucose and triglycerides as surrogate for identifying insulin resistance in apparently healthy subjects. Metab Syndr Relat Disord. 2008;6:299-304.

14. Khan SH, Sobia F, Niazi NK, Manzoor SM, Fazal N, Ahmad F. Metabolic clustering of risk factors: evaluation of triglyceride-glucose index (TyG index) for evaluation of insulin resistance. Diabetol Metab Syndr. 2018;10:74.

15. Guerrero-Romero F, Simental-Mendía LE, González-Ortiz M, MartínezAbundis E, Ramos-Zavala MG, Hernández-González SO, et al. The product of triglycerides and glucose, a simple measure of insulin sensitivity. Comparison with the euglycemic-hyperinsulinemic clamp. J Clin Endocrinol Metab. 2010;95:3347-51.

16. Sánchez-Înigo L, Navarro-González D, Fernández-Montero A, PastranaDelgado J, Martínez JA. The TyG index may predict the development of cardiovascular events. Eur J Clin Invest. 2016;46:189-97.

17. Lambrinoudaki I, Kazani MV, Armeni E, Georgiopoulos G, Tampakis K, Rizos D, et al. The TyG index as a marker of subclinical atherosclerosis and arterial stiffness in lean and overweight postmenopausal women. Heart Lung Circ. 2018;27:716-24

18. Park GM, Cho YR, Won KB, et al. Triglyceride glucose index is a useful marker for predicting subclinical coronary artery disease in the absence of traditional risk factors. Lipids Health Dis. 2020;19:7.

19. Cho YR, Ann SH, Won KB, et al. Association between insulin resistance, hyperglycemia, and coronary artery disease according to the presence of diabetes. Sci Rep. 2019;9:6129.

20. Van Minh H, Ng N, Juvekar S, Razzaque A, Ashraf A, Hadi A, et al. Selfreported prevalence of chronic diseases and their relation to selected sociodemographic variables: a study in INDEPTH Asian sites, 2005. Prev Chronic Dis. 2008:5:A86

21. American Diabetes Association. Classification and diagnosis of diabetes: standards of medical care in diabetes-2019. Diabetes Care. 2019;42(Suppl 1):S13-S28

22. Matthews DR, Hosker JP, Rudenski AS, Naylor BA, Treacher DF, Turner RC. Homeostasis model assessment: insulin resistance and beta-cell function from fasting plasma glucose and insulin concentrations in man. Diabetologia. 1985;28:412-9.

23. National Cholesterol Education Program (NCEP) Expert Panel on Detection, Evaluation, and Treatment of High Blood Cholesterol in Adults (Adult Treatment Panel III). Third Report of the National Cholesterol Education Program (NCEP) Expert Panel on Detection, Evaluation, and Treatment of High Blood Cholesterol in Adults (Adult Treatment Panel III) final report. Circulation. 2002;106:3143-421.

24. Leipsic J, Abbara S, Achenbach S, et al. SCCT guidelines for the interpretation and reporting of coronary CT angiography: a report of the Society of Cardiovascular Computed Tomography Guidelines Committee. J Cardiovasc Comput Tomogr. 2014:8:342-58.

25. Vasques AC, Novaes FS, de Oliveira MS, Souza JR, Yamanaka A, Pareja $J$, et al. TyG index performs better than HOMA in a Brazilian population: a hyperglycemic clamp validated study. Diabetes Res Clin Pract. 2011;93:e98-e100.

26. Kim MK, Ahn CW, Kang S, Nam JS, Kim KR, Park JS. Relationship between the triglyceride glucose index and coronary artery calcification in Korean adults. Cardiovasc Diabetol. 2017;16:108.

27. Navarro-González D, Sánchez-Iñigo L, Fernández-Montero A, PastranaDelgado J, Martinez JA. TyG index change is more determinant for forecasting type 2 diabetes onset than weight gain. Medicine. 2016;95:e3646.

28. Zhang M, Wang B, Liu Y, Sun X, Luo X, Wang C, et al. Cumulative increased risk of incident type 2 diabetes mellitus with increasing triglyceride glucose index in normal-weight people: the Rural Chinese Cohort Study. Cardiovasc Diabetol. 2017;16:30.

29. Brahimaj A, Rivadeneira F, Muka T, Sijbrands EJG, Franco OH, Dehghan A, Kavousi M. Novel metabolic indices and incident type 2 diabetes among women and men: the Rotterdam Study. Diabetologia. 2019;6:1581-90.
30. Jin JL, Cao YX, Wu LG, You XD, Guo YL, Wu NQ, et al. Triglyceride glucose index for predicting cardiovascular outcomes in patients with coronary artery disease. JThorac Dis. 2018;10:6137-46.

31. Jin JL, Sun D, Cao YX, Guo YL, Wu NQ, Zhu CG, et al. Triglyceride glucose and haemoglobin glycation index for predicting outcomes in diabetes patients with new-onset, stable coronary artery disease: a nested casecontrol study. Ann Med. 2018;50:576-86.

32. Mao Q, Zhou D, Li Y, Wang Y, Xu SC, Zhao XH. The triglyceride-glucose index predicts coronary artery disease severity and cardiovascular outcomes in patients with non-ST-segment elevation acute coronary syndrome. Dis Markers. 2019;2019:6891537.

33. Luo E, Wang D, Yan G, Qiao Y, Liu B, Hou J, Tang C. High triglycerideglucose index is associated with poor prognosis in patients with acute ST-elevation myocardial infarction after percutaneous coronary intervention. Cardiovasc Diabetol. 2019;18:150.

34. Irace C, Carallo C, Scavelli FB, De Franceschi MS, Esposito T, Tripolino C, Gnasso A. Markers of insulin resistance and carotid atherosclerosis. A comparison of the homeostasis model assessment and triglyceride glucose index. Int J Clin Pract. 2013;67:665-72.

35. Lee SB, Ahn CW, Lee BK, et al. Association between triglyceride glucose index and arterial stiffness in Korean adults. Cardiovasc Diabetol. 2018;17:41.

36. Won KB, Park GM, Lee SE, et al. Relationship of insulin resistance estimated by triglyceride glucose index to arterial stiffness. Lipids Health Dis. 2018;17:268.

37. Park K, Ahn CW, Lee SB, et al. Elevated TyG index predicts progression of coronary artery calcification. Diabetes Care. 2019:42:1569-73.

38. Nguyen HL, Nguyen QN, Ha DA, Phan DT, Nguyen NH, Goldberg RJ. Prevalence of comorbidities and their impact on hospital management and short-term outcomes in Vietnamese patients hospitalized with a first acute myocardial infarction. PLoS ONE. 2014;9:e108998.

39. Nguyen T, Le KK, Cao HTK, Tran DTT, Ho LM, Thai TND, et al. Association between in-hospital guideline adherence and postdischarge major adverse outcomes of patients with acute coronary syndrome in Vietnam: a prospective cohort study. BMJ Open. 2017;7:e017008.

40. Valensi P, Prévost G, Schnell O, Standl E, Ceriello A. Targets for blood glucose: what have the trials told us. Eur J Prev Cardiol. 2019;26(2 suppl):64-72.

41. Lawler PR, Kotri G, Koh M, et al. Real-world risk of cardiovascular outcomes associated with hypertriglyceridaemia among individuals with atherosclerotic cardiovascular disease and potential eligibility for emerging therapies. Eur Heart J. 2020;41:86-94.

42. Boekholdt SM, Arsenault BJ, Mora S, et al. Association of LDL cholesterol, non-HDL cholesterol, and apolipoprotein B levels with risk of cardiovascular events among patients treated with statins: a meta-analysis. JAMA. 2012:307:1302-9.

43. Fruchart JC, Davignon J, Hermans MP, et al. Residual macrovascular risk in 2013: what have we learned? Cardiovasc Diabetol. 2014;13:26.

44. Valensi P, Avignon A, Sultan A, Chanu B, Nguyen MT, Cosson E. Atherogenic dyslipidemia and risk of silent coronary artery disease in asymptomatic patients with type 2 diabetes: a cross-sectional study. Cardiovasc Diabetol. 2016;15:104.

45. Borén J, Williams KJ. The central role of arterial retention of cholesterolrich apolipoprotein-B-containing lipoproteins in the pathogenesis of atherosclerosis: a triumph of simplicity. Curr Opin Lipidol. 2016;27:473-83.

46. Mach F, Baigent C, Catapano AL, et al. 2019 ESC/EAS Guidelines for the management of dyslipidaemias: lipid modification to reduce cardiovascular risk. Eur Heart J. 2020:41:111-88.

47. Budoff M. Triglycerides and triglyceride-rich lipoproteins in the causal pathway of cardiovascular disease. Am J Cardiol. 2016;118:138-45.

48. Hermans MP, Valensi P. Elevated triglycerides and low high-density lipoprotein cholesterol level as marker of very high risk in type 2 diabetes. Curr Opin Endocrinol Diabetes Obes. 2018;25:118-29.

49. Bhatt DL, Steg PG, Miller M, for the REDUCE-IT Investigators, et al. Cardiovascular risk reduction with icosapent ethyl for hypertriglyceridemia. $\mathrm{N}$ Engl J Med. 2019;380:11-22.

\section{Publisher's Note}

Springer Nature remains neutral with regard to jurisdictional claims in published maps and institutional affiliations. 